\title{
Empresas de Arqueología y Arqueología urbana: investigación, negocio, profesión
}

\author{
R.Ma Domínguez Alonso* \\ A. Fernández Ugalde* \\ J.L. Herce Yuste* \\ M. Menasanch de Tobaruela* \\ M.Ma Presas Vías*
}

El asunto que se argumenta en esta comunicación resulta más adecuado para un debate abierto que para su exposición extensa. En consecuencia, seremos breves, y nos limitaremos a presentar una serie de puntos referentes a la situación actual, enfocados desde la óptica de nuestra propia experiencia y, por tanto, con especial atención al caso de Madrid, y a plantear algunas propuestas que, en nuestra opinión, pueden ser de utilidad para, al menos, mejorar algunos aspectos de un sistema de funcionamiento todavía poco articulado.

\section{INTRODUCCIÓN}

En los últimos años, como resultado de la coincidencia de la expansión de la economía de libre mercado, del desarrollo acelerado de infraestructuras y construcciones y de un mayor compromiso de las administraciones autonómicas en la conservación y documentación del patrimonio histórico, se han multiplicado en España las intervenciones denominadas de urgencia, sobre todo en los núcleos urbanos. Como consecuencia, ha surgido la necesidad de arqueólogos que se encarguen de documentar las intervenciones potencialmente destructivas y de rescatar el máximo de información antes de que ésta se pierda irreversiblemente. Dado que los programas de la Administración competente no plantean la necesidad de mantener un cuerpo permanente de arqueólogos (cuyo número depende en buena medida del volumen de actividad que desarrollen las iniciativas de construcción pública y privada en un momento dado), en Madrid, y cada vez más también en otras ciudades, se ha optado por recurrir ampliamente a profesionales no insertos en organismos públicos. Es decir, si en apariencia el nacimiento de las empresas se debe a causas coyunturales como el desarrollo de la arqueología de intervención y de la arqueología urbana y la carencia de arqueólogos dedicados a intervenciones de campo por parte de la administración, en el fondo subyace una situación estructural: no se plantea incrementar los cuerpos de arqueólogos por parte de la Administración competente o, en el caso de incrementarlos, su ocupación prioritaria sería la de gestión o conservación del patrimonio histórico, mientras que la ejecución de las intervenciones la seguirán desarrollando profesionales privados.

Las empresas de arqueología, pues, no son sino el último extremo del sistema aunque, como hemos dicho, forman parte estructural de él, y sus miembros carezcan de responsabilidad directa en la organización del actual status quo. Sin embargo, los arqueólogos profesionales' desempeñamos un papel activo en la aplicación de una política de gestión del patrimonio generada desde las instituciones competentes y participamos en una situación que creemos criticable y mejorable.

Hasta el momento, uno de los terrenos de mayor implantación de las empresas de arqueología lo constituye la arqueología urbana o, estrictamente, las intervenciones arqueológicas de urgencia en núcleos urbanos. Esto supone que a los problemas generales de la política y de la gestión del patrimonio histórico se sumen los derivados de la incorporación de profesionales en libre ejercicio y de empresas.

Probablemente, la arqueología urbana sea el 
campo de la arqueología en el que interviene un mayor número de instancias diversas: administración autonómica, administración municipal, organismos de investigación, agentes de construcción privados y profesionales en libre ejercicio o asociados en empresas. En consecuencia, en cada ciudad en concreto se han generado situaciones diversas, según el grado de actuación de los elementos que intervengan y las relaciones que se establezcan entre ellos, y tales situaciones pueden variar mucho de unos núcleos urbanos a otros. De hecho, esta hetereogeneidad, por llamarle de alguna manera, es una de las características de la arqueología urbana, con las consecuencias que pueden tener, entre otras cosas, para el desarrollo de una historia urbana global. A este respecto nos interesa también destacar que, como integrantes de una empresa, hablamos desde un punto de vista peculiar, distinto al institucional, pero seguramente también alejado de la visión de los profesionales de otras empresas de arqueología: otra característica de la arqueología urbana con participación de profesionales privados es que a la fragmentación entre los distintos núcleos urbanos se añade la hetereogeneidad que se puede originar dentro de un mismo núcleo cuando en él intervienen arqueólogos que actúan con criterios diferentes. Sobre esta situación, que en Madrid tal vez esté especialmente marcada, insistiremos a lo largo de la comunicación.

En las ciudades, la arqueología medieval y moderna son probablemente las que más sufren las consecuencias de una gestión y una intervención defectuosas, bien porque la mayor parte de la estratificación de valor arqueológico corresponde a estos periodos, bien porque, en caso de existir periodos subyacentes de mayor monumentalidad, éstos son objeto preferente de la atención institucional y de la investigación. Recíprocamente, la arqueología de época medieval y moderna puede resultar la más beneficiada por la multiplicación de las intervenciones siempre que éstas se engloben en un programa adecuado.

\section{EMPRESAS DE ARQUEOLOGÍA $Y$ ARQUEOLOGÍA URBANA: SITUACIÓN ACTUAL.}

Después de esta breve introducción, en la que hemos pretendido plantear algunos puntos genera- les, pasaremos a exponer cuál ha sido el origen del sistema de funcionamiento en Madrid y cuál es su situación actual.

A partir de mediados de los años 80, coinciden la transferencia de competencias en materia de cultura a la Comunidad de Madrid y una explosión constructiva en la ciudad. En consecuencia, a partir de ese momento a los problemas propios del planeamiento urbano se añaden nuevos problemas derivados esencialmente de la confrontación de intereses de especulación y construcción con la necesidad de documentación y conservación del patrimonio. Ante esta nueva situación, la administración de cultura toma dos medidas esenciales:

-Por una parte, se delimitan una serie de zonas protegidas amplias que se declaran BIC con categoría de zona arqueológica y/o paleontológica, en las que toda iniciativa de construcción o de rehabilitación debe ir precedida por la ejecución de un peritaje arqueológico y la emisión de un dictamen, sin los cuales no es posible obtener la preceptiva licencia municipal de obra. Es el caso, en Madrid, del casco histórico comprendido en el interior de los recintos amurallados del siglo IX y del siglo XI-XII, y de la zona comprendida en el interior de la cerca de Felipe IV3; además, están declarados como BIC parte de los cascos urbanos de Alcalá de Henares, Aranjuez, Getafe y otras ciudades o pueblos de la Comunidad.

-Por otra parte, y ésta es la mayor innovación del sistema vigente en Madrid, se establece de forma oficiosa un procedimiento que promueve la financiación de las intervenciones arqueológicas por los promotores que realizan obras en tales espacios protegidos (ante la alternativa de la financiación por parte de la Consejería de Educación y Cultura, sometida a la lentitud de los trámites administrativos, los constructores prefieren, sistemáticamente, hacerse cargo de los costes).

Citaremos, al margen, que las intervenciones programadas, financiadas directamente por la Comunidad de Madrid, se circunscriben, casi exclusivamente, a ámbitos extraurbanos (con la excepción de algunas intervenciones en Alcalá de Henares).

A partir del amplio desarrollo de la construcción urbana y de esta nueva normativa surge, como decí- 
amos en la introducción, la necesidad de contar con un buen número de arqueólogos que hagan posible llevar a la práctica la normativa legal. Ante esto, la Comunidad de Madrid ha optado por encomendar la realización de las intervenciones a profesionales privados. En un principio, en Madrid primó la figura del arqueólogo como trabajador autónomo. Sin embargo, por las propias características del trabajo arqueológico, muchos profesionales fueron constituyendo equipos estables que, con el tiempo, han derivado en la constitución de empresas bajo diversas formas jurídicas, fundamentalmente comunidades de bienes, sociedades limitadas o, en nuestro caso, sociedad cooperativa. Entre las razones que han promovido la constitución de empresas se encuentran la necesidad de establecer relaciones contractuales entre empresas constructoras y arqueólogos y, a menudo, las propias exigencias de la administración pública en lo referente a concursos, contrataciones, etc.

En este punto se plantea la cuestión de la "asignación" de los trabajos a los arqueólogos o grupos de arqueólogos. Para resolverla, los propios técnicos de la Consejería de Educación y Cultura impulsan la creación, dentro del Colegio de Doctores y Licenciados, de una Comisión de Arqueólogos que, entre otras funciones, se ocupe de poner en marcha una bolsa de trabajo a la que, al menos en teoría, puedan acceder todos los arqueólogos colegiados. Se optó por elaborar, atendiendo a los curricula, un listado por "ámbitos de especialización" un tanto pintorescos ("arqueología urbana" vs. "arqueología de campo", "prospección", etc.) y por períodos históricos (de Paleolítico a Moderno). Así, cuando un promotor se ve en la necesidad de contratar a un técnico y acude a la Consejería de Educación y Cultura, se le enuncia el perfil del arqueólogo (por ejemplo, "arqueología urbana" o "Paleolítico") y se le remite al CDL y a su bolsa de trabajo. El promotor se pone entonces en contacto con una serie de arqueólogos a los que solicita un presupuesto para la intervención de que se trate y, en función de este presupuesto, elige al que le proporcione las mejores condiciones económicas y plazos de ejecución. Una vez establecido un acuerdo promotor-arqueólogo, el técnico o los técnicos que vayan a realizar la intervención elaboran un proyecto que presentan a los técnicos de la Comunidad de Madrid para que, en su caso, le den el visto bueno. Si éstos consideran que el proyecto se ade- cúa a las características arqueológicas del solar, extienden un permiso de excavación nominal y, a partir de ese momento, el arqueólogo actúa en delegación de la Administración competente en materia de patrimonio. Concluída la intervención, el arqueólogo emite un informe, positivo o negativo, que remite a los técnicos de la Comunidad de Madrid. Éstos son, en último término, los que deben otorgar el dictamen para que el proyecto de construcción siga adelante, o bien, si lo consideran necesario, advertir que antes de iniciar la construcción es obligatorio ampliar la intervención arqueológica. La correspondiente ampliación de presupuesto la negocia el profesional independiente con el promotor.

A grandes rasgos, este es el sistema de funcionamiento vigente en Madrid desde 1985. A raíz de su puesta en práctica, probablemente Madrid sea una de las ciudades de nuestro país en las que se ha realizado un mayor número de intervenciones arqueológicas en los últimos años, lo cual, por sí mismo, no implica un desarrollo paralelo del conocimiento histórico de la ciudad.

¿Cuáles son, desde nuestro punto de vista, los aspectos positivos de este sistema?

En primer lugar, la normativa de patrimonio histórico en Madrid es, probablemente, una de las más exigentes en cuanto a la obligatoriedad de intervención y la amplitud de las zonas protegidas, y quizá la que logra trasvasar mayor volumen de fondos privados en beneficio, al menos teórico, de la documentación y la conservación del patrimonio.

Asimismo, la multiplicación de las intervenciones ha permitido que se preste atención a aspectos que, por poco gratificantes o espectaculares, antes estaban abandonados a pesar de su valor histórico (arqueología moderna y, en general, la de todos los períodos medievales post-islámicos).

Por otra parte, la generalización de la participación de profesionales privados en la arqueología urbana ha hecho posible la creación de puestos de trabajo que pueden permitir la promoción profesional y científica de licenciados que, de otro modo, tal vez se hubiesen visto obligados a abandonar su profesión o buscar otras salidas laborales. 
Por último, consideramos que este sistema puede favorecer la integración de los arqueólogos en la actividad económica, una mayor difusión del valor del patrimonio histórico, y una estimación más justa del profesional de la arqueología ante otros profesionales y ante la sociedad en general.

Ahora bien, frente a estos aspectos positivos, el sistema que se está aplicando en Madrid presenta también una serie de carencias notables que han dado lugar a una situación cada vez más alejada del objetivo primario de proteger el patrimonio y profundizar en el conocimiento histórico de la ciudad. Entre las limitaciones que consideramos de consecuencias más graves podemos citar:

-la ausencia de un programa global que articule las intervenciones puntuales, ausencia que ha mermado los potenciales aspectos positivos del aumento numérico de las intervenciones;

-la escasa intervención de la administración competente una vez puesto en marcha el sistema. Ante la extraordinaria proliferación de intervenciones arqueológicas, la supervisión de la Consejería de Educación y Cultura se limita en la mayoría de los casos al mero trámite de concesión de permisos y recepción de memorias de excavación. Como consecuencia de esta actitud por parte de la administración, el funcionamiento del sistema se ha ido haciendo cada vez más liberal (en el sentido de ausencia de control), y la mayor parte del proceso de intervención en el patrimonio cultural ha quedado al albedrío de los particulares, representados por los promotores de obra y las empresas de arqueología o los profesionales en libre ejercicio (con la intervención del CDL a través de sus listados de profesionales). Sólo muy recientemente la Consejería de Educación y Cultura ha empezado a fijar algunos criterios previos para las intervenciones, esencialmente en lo referido a extensión mínima del espacio excavado en cada inmueble;

-la falta de criterios de selección del arqueólogo adecuado para cada intervención o, lo que es lo mismo, indefinición del perfil del profesional que ejecuta las intervenciones arqueológicas urbanas de urgencia, más allá de su vaga inscripción en un listado basado en una experiencia conferida por el número de intervenciones dirigidas.
Estas carencias existen desde el origen del llamado "sistema Madrid" y, tras ocho años de experiencia, se siguen manteniendo. De hecho, otra limitación, aunque en este caso no exclusivamente achacable a los organizadores del sistema, es la casi total ausencia de debate acerca del funcionamiento y de los resultados obtenidos en todo este tiempo. En ocho años no se han convocado ningún tipo de jornadas, mesas redondas o reuniones en las que se haya planteado para su discusión los diversos aspectos y resultados del llamado "sistema Madrid", y en las que podrían tener cabida representantes de todas las instancias implicadas y también de las no implicadas, o que hasta el momento no han estimado oportuno implicarse, como son las universidades, el CSIC o las diversas asociaciones de arqueólogos profesionales. La única iniciativa en este sentido ha sido la realización de dos exposiciones, una acerca de la historia de la arqueología madrileña y otra acerca del Madrid islámico, pero ninguna de las dos ha ido acompañada de algún tipo de conferencia o mesa redonda abierta a la discusión. En cuanto a las publicaciones de excavaciones urbanas, son todavía escasas, y sólo contienen un resumen de los resultados obtenidos.

Ante las carencias propias del sistema a las que hemos hecho alusión, planteamos, en primer lugar, que es esencial que las instituciones públicas hagan efectiva su labor de tutela puesto que, de lo contrario, la función de salvaguardar el patrimonio recae sobre los profesionales privados que llevan a cabo la intervención. De esta manera, la contradicción entre los intereses de los agentes privados que ejecutan obras y que, según las leyes vigentes, están obligados a llevar a cabo intervenciones de urgencia previas, y el interés social de protección y acrecentamiento del patrimonio histórico público deberá resolverla el profesional independiente, tratando, además, con aquéllos que le están otorgando los fondos y constituyen parte de sus clientes. Si las instituciones competentes no hacen efectivo su deber de tutela, más allá de la mera concesión de permisos a profesionales y de una más o menos somera supervisión de los proyectos de intervención y de sus resultados, a la postre se entrega el patrimonio a los particulares, con todos los riesgos que esto implica.

Sin una gestión efectiva y eficaz, la calidad de la intervención desciende cada vez más: en una activi- 
dad sometida a la petición de ofertas por el promotor de la obra, la introducción de profesionales con escasos escrúpulos que aceptan que su labor se limite a cubrir los intereses del promotor produce una degradación de la competencia y, por tanto, la tendencia del sistema a convertirse prácticamente en un mercado de venta de dictámenes a bajo precio. Dicho de otra manera, existe una contradicción entre beneficio del arqueólogo o la empresa y minuciosidad y calidad de la intervención arqueológica que sólo puede minimizarse mediante una gestión efectiva de la administración competente.

En el caso de Madrid, esta situación se ve aún más favorecida por el hecho de que los restos que se conservan dentro del casco urbano, en su gran mayoría, y si excluímos los tramos de muralla, carecen de monumentalidad, lo que facilita que se puedan destruir sin que ello tenga mayor resonancia. Esta situación se puede hacer extensible a otras ciudades, y en muchos casos afecta en particular a las fases medievales de las que, en concreto en Madrid, rara vez se conservan estructuras arquitectónicas.

En definitiva, la insuficiente o, cuando menos, escasa capacidad de control de la administración en la arqueología urbana madrileña es lo que permite que las empresas constructoras presionen al arqueólogo, a espaldas de la Consejería de Educación y Cultura, para reducir la intervención incluso a los mínimos para cubrir el expediente. En nuestra opinión, éste es el verdadero problema de lo que se ha dado en denominar arqueología profesional, y el talón de Aquiles de sistemas teóricamente muy avanzados, como el establecido en Madrid. La cuestión estriba en confundir financiación privada con gestión o funcionamiento liberal de las intervenciones, lo cual favorece el desarrollo de una competencia salvaje entre empresas de arqueología, con ventaja para aquéllas con menos escrúpulos. Los problemas se agudizan cuando en la competencia se introducen empresas creadas con el mínimo de requisitos legales, con el objetivo de aprovechar al máximo la coyuntura de negocio que permiten el boom constructivo y una normativa de patrimonio más exigente. Estas empresas, que cuando adquieren un volumen de negocio suficiente recurren a la contratación de personal al mínimo costo (generalmente, recién licenciados o licenciados en paro), poseen una estructura netamente vertical, y extien- den relaciones laborales empresario-asalariado en las que el profesional contratado temporalmente sólo aporta su fuerza de trabajo, se convierte en un elemento del que se obtiene beneficio ${ }^{4}$ y queda alienado como arqueólogo, puesto que no elabora el proyecto, ni interviene en el planteamiento de la intervención, ni participa en el estudio o en su publicación. En definitiva, se generalizan modos de proceder en los que la condición del arqueólogo y la calidad de la labor de intervención arqueológica tienden a resentirse.

En diversas ocasiones, colectivos de profesionales o profesionales independientes han defendido y defienden la elaboración de un código deontológico como el instrumento básico para resolver la situación que se crea cuando la financiación es privada y el control de la administración no es efectivo5. Sin embargo, creemos que, en último término, confiar en el respeto a este código significa dejar en manos de la ética de privados el tratamiento correcto de algo que es patrimonio público, lo cual, como venimos diciendo, es una evidente contradicción. Con esto no queremos decir que el código deontológico no sea necesario, pero éste no se llevará a la práctica sin un control efectivo. Por otra parte, este código no llegará a resolver otros problemas, como es el de obtener beneficio a base de la simple explotación de la fuerza de trabajo. Pero, en todo caso, creemos que éste es un problema irresoluble, ya que, dentro de una economía de libre mercado, no es posible mantener ningún control efectivo sobre este tipo de actuación, a no ser que se pase por una organización de tipo sindical.

\section{EMPRESAS DE ARQUEOLOGÍA ¿NEGO- CIO? ¿VIABILIDAD?}

Resulta obvio que quien se lanza a la creación de una empresa posee algún tipo de expectativas de viabilidad económica y de crecimiento sostenido. La cuestión clave es cómo se conciba este "negocio". Nosotros entendemos por negocio una actividad económica que permita, a medio o largo plazo, llegar a ingresar más de lo que se gasta, descontados unos ingresos dignos, de manera que la empresa se pueda seguir manteniendo. En definitiva, es una concepción opuesta a la de obtención de un beneficio rápido a base del deterioro de la cali- 
dad del trabajo, situación que en nuestro sector es todavía más grave, puesto que lo que sufre las consecuencias de este deterioro es patrimonio público.

Resumimos sumariamente las razones que nos han llevado a optar, a pesar de su mayor rigidez y del mayor costo comparativo de las cuotas sociales, por una estructura de empresa cooperativa expuestas recientemente en la RAT de Santiago de Compostela6: estructura asamblearia e igualitaria, predominio del capital/trabajo y concepción de trabajo en equipo, por oposición a estructura vertical, con predominio del capital y establecimiento de relaciones empresario-asalariado.

En todo caso, por lo que respecta a nuestra cooperativa y a otras muchas empresas similares a la nuestra, todavía está por demostrar que estos proyectos sean viables y, aún más, que de ellos se pueda extraer beneficio, puesto que el principal coste que debe cubrirse son los propios ingresos de los trabajadores, y muchas empresas estamos todavía lejos de alcanzar unos salarios, no ya equiparables a los de otros profesionales de nuestra misma cualificación, sino lo suficientemente dignos y estables como para vivir de nuestro trabajo como arqueólogos.

\section{NUESTRA PROPUESTA DE SISTEMA.}

Después de exponer un ejemplo de funcionamiento y nuestra postura como cooperativa ante la situación que se plantea al introducir ampliamente en la intervención sobre el patrimonio histórico la actividad de empresas de arqueología, pasaremos a exponer, desde nuestra perspectiva de profesionales, algunas propuestas referentes a aspectos concretos que creemos pueden ser objeto de mejora.

En primer lugar, ante la ausencia de un programa global que articule las intervenciones puntuales, recomendamos la necesidad de incluir planes directores de la actividad arqueológica urbana en los planes generales de ordenación, planes en cuya elaboración deben tomar parte activa todos los arqueólogos que participan en la actividad arqueológica de las ciudades. ¿Qué debe contener un programa de intervención? Naturalmente, no sólo la obligatoriedad de efectuar intervenciones arqueológicas de urgencia previas a las actividades constructivas en espacios protegidos, sino unas líneas de investigación que contemplen la necesidad de intervenciones arqueológicas programadas a la hora de profundizar en el conocimiento histórico de la ciudad y que, de hecho, serían las que vendrían a establecer las directrices del programa de intervenciones.

Junto a estas intervenciones programadas podría desarrollarse otra línea destinada a conciliar los diferentes intereses que se enfrentan en una ciudad (es decir, intereses de los promotores, de los privados que demandan una vivienda o nuevos aparcamientos, e intereses sociales de conservación del patrimonio), ajustando lo más posible el alcance de la intervención a las características de la zona o de la parcela afectada. Nos referimos, en concreto, a la puesta en práctica de metodologías de valoración previa de la potencialidad estratigráfica en núcleos habitados, ya experimentadas con éxito en ciudades de Inglaterra, Francia o Italia, y que, partiendo de procedimientos empíricos que no se limitan a la consulta de fuentes no estrictamente arqueológicas, analizan las características del subsuelo (análisis de la topografía del terreno, prospecciones no destructivas, sondeos selectivos para constatar los resultados obtenidos por otros medios, etc), de manera que se pueda prever la potencia estratigráfica conservada y, por tanto, programar racionalmente las actuaciones arqueológicas y delimitar espacios de reserva.

Por otra parte, proyectos de este tipo harían posible la colaboración entre distintas instancias implicadas en el conocimiento y la conservación del patrimonio: universidad, CSIC, administración y profesionales que, hoy por hoy, y al menos en Madrid, se encuentran muy distanciados, y cuya relación parece que se limita a dirigirse críticas mutuas off the record, pero nunca a plantear posibles vías de colaboración. Quizá este enfoque contribuyera a resolver esa dicotomía actual entre investigadores (con toda la carga de abstracción, de ambiciosos proyectos y de "pureza" que connota el término) y técnicos (con toda su carga de "auxiliares" de los "hombres de ciencia", de ausencia de planteamientos epistemológicos y de positivismo inconsciente), que consideramos errónea.

Uno de los problemas para la puesta en marcha de este tipo de programas sería su financiación. Puesto que en el actual estado de cosas vemos diff- 
cil que corriese a cargo de la propia Administración, ésta podría promover el patrocinio de empresas o fundaciones. En cualquier caso, hemos mantenido en otro lugar que la financiación privada de las intervenciones es, en sí misma, una medida progresista, salvando los riesgos que se derivan de la laxitud de la supervisión de la Administración competente y del abandono de los arqueólogos a las presiones de los promotores?. No parece legítimo que las instituciones públicas tengan que asumir los costes derivados de la necesidad de salvaguardar el patrimonio de las actividades de construcción promovidas por particulares sólo para evitar los riesgos de contaminación por la iniciativa privada. Pensamos que la fijación de normas de procedimiento, requisitos de intervención, pautas de financiación y códigos de compromiso entre arqueólogos y promotores pueden bastar para fijar la buena marcha de un sistema en el que al promotor de una obra se le exige hacer frente a los compromisos y costes añadidos de trabajar en un espacio arqueológico protegido8.

El carácter que deba adquirir la financiación es, con todo, uno de los aspectos de mayor polémica de nuestra actividad, como puede apreciarse en cualquiera de las recientes reuniones, encuentros y coloquios en los que se ha tratado de la arqueología profesional o de intervención. Sin despreciar las ventajas que se derivan de la canalización de fondos de las empresas promotoras de obras puntuales, una solución viable puede ser su combinación con fórmulas de patrocinio de los programas generales por empresas o fundaciones, promovidos por la administración de cultura, toda vez que no parece que corran vientos favorables a la extensión de las cargas fiscales generales (con vistas a la financiación de actividades culturales) sobre la empresa privada, hoy considerada motor y guía del crecimiento económico...

Con respecto a la necesidad de control efectivo por parte de la Administración competente, sobre la que venimos insistiendo a lo largo de nuestra intervención, ésta debe extender sus competencias mucho más allá de la simple concesión de permisos y del mero archivo de la documentación generada. ¿Cuáles son nuestras propuestas a este respecto? En primer lugar, y mientras no se establezcan otros mecanismos de control más operativos, consideramos esencial incrementar y hacer efectiva la inspec- ción por parte de los técnicos de la administración. Ahora bien, hay que señalar que somos conscientes de las dificultades con que estos técnicos se encuentran a la hora de realizar inspecciones, dificultades que suelen basarse fundamentalmente en la falta de personal que pueda dedicarse a estas tareas sin descuidar otras. Por otra parte, consideramos imprescindible que las Consejerías de Cultura establezcan pliegos de condiciones con requisitos mínimos para toda intervención, normativas que homogeneícen las metodologías (que, a su vez, estarían supeditadas a los objetivos fijados en un programa global) y sistemas de registro de la información (hasta llegar a exigir el empleo de procedimientos de excavación estratigráica9 ${ }^{9}$ ) y que se fijen canales para la publicación de los resultados obtenidos, publicación que debería tener carácter íntegro y obligatorio.

Hacemos hincapié en la necesidad de un control efectivo de las excavaciones financiadas por los promotores puesto que el interés prioritario de éstos no es, obviamente, la conservación y documentación del patrimonio, sino la economía y rapidez de los trabajos, y porque la experiencia madrileña demuestra que un funcionamiento totalmente liberalizado en la práctica, favorece la actividad de arqueólogos oportunistas. Asímismo, otro aspecto que consideramos debe ser promovido desde la administración es la difusión social de los resultados de las intervenciones de rescate mediante la publicación de las memorias, la divulgación didáctica y la organización de exposiciones periódicas en las que, además de los resultados de las actuaciones arqueológicas se diese a conocer el volumen de fondos invertidos, etc., de forma que la utilidad de las intervenciones de urgencia quede manifiesta para todos los ciudadanos y, cada vez más, para las propias empresas que están obligadas a financiarlas.

Por último, con respecto a la cuestión de qué arqueólogos pueden dirigir intervenciones urbanas, desde nuestro punto de vista es obvio que en la situación actual se plantean diversos problemas. Es decir, puesto que en las actuales circunstancias existe una demanda objetiva de arqueólogos de intervención profesionales, y puesto que las competencias sobre el patrimonio histórico corresponden a las administraciones autonómicas de cultura, que son las que conceden la dirección de un proyecto por delegación, ia quién corresponde valorar la 
cualificación profesional del arqueólogo que solicita un permiso de intervención determinado, cuando los únicos requisitos exigibles a un técnico son su licenciatura universitaria en prehistoria y/o arqueología, la elaboración de un proyecto de intervención adecuado y el cumplimiento de las condiciones establecidas por la administración?

En nuestra opinión, si existe la posibilidad de que licenciados incapaces lleguen a dirigir intervenciones, se debe a que, hasta el momento, la Universidad, al menos en el caso de Madrid, no está respondiendo a las exigencias sociales y no proporciona ni una buena formación histórica, ni una preparación adecuada para el trabajo de campo. La solución, en cualquier caso, no pasa por que los técnicos de las Consejerías de Cultura patrimonialicen la concesión de permisos de intervención, otorgándolos a quien ellos consideren capacitado. Cuando la arqueología se convierte en una actividad profesional, en numerosas ocasiones financiada por particulares, y que constituye la única fuente de ingresos para muchos arqueólogos, es necesario hacer explícitos los baremos y los requisitos exigibles a los arqueólogos, de forma que el único condicionante sea la calidad de la intervención, y no la afinidad a aquéllos que poseen la competencia legal de la concesión de permisos.

Desde luego, lo que tampoco nos parece adecuado es la solución por la que se ha optado en Madrid, donde el perfil del técnico lo define la comisión de arqueólogos del CDL, que ha establecido unos baremos a partir de los cuáles, y previa presentación de curriculum por parte de los colegiados que deseen acceder a la bolsa de trabajo, es la comisión del CDL la que decide quién es especialista en determinado periodo cultural o tipo de intervención 10 y, por tanto, qué individuos pueden estar incluídos en los listados que se entregan a los promotores y constructores cuando éstos acuden al Colegio en busca de técnicos.

Quizás, el perfil del arqueólogo que interviene en un núcleo urbano se defina esencialmente por su participación activa en los debates y problemas de la historia urbana. En un ambiente urbano pluriestratificado, sin embargo, la situación ideal es que la intervención se desarrolle por un equipo integrado por arqueólogos especializados en distintos períodos históricos o en los diversos aspectos del regis- tro arqueológico (paleocarpólogos, arqueozoólogos, etc.), todos ellos con una formación adecuada para el análisis de la estratigrafía arqueológica urbana. Sin embargo, esta concepción de trabajo en equipo choca frontalmente con la conjunción de financiación privada y de competitividad con escasos escrúpulos, en la que se tiende a cubrir sólo los gastos y condiciones estrictamente necesarios para obtener el dictamen que exige la normativa vigente. En consecuencia, se pone de nuevo de manifiesto la necesidad de una intervención efectiva de la administración competente para garantizar una mayor calidad del trabajo.

\section{A MODO DE CONCLUSIÓN}

A no ser que pretendamos poner en duda el propio sistema global de economía capitalista de mercado y la normativa vigente de protección de los bienes culturales, promoviendo modelos alternativos, no tiene sentido discutir la aplicación de fondos privados a la documentación y conservación del patrimonio. La mediatización de la actividad de construcción por la arqueología es una conquista indiscutible: entre sus consecuencias cabe citar la ampliación del volumen de información histórica que se puede poner a disposición de los investigadores y la integración de la figura del arqueólogo en la actividad económica y en la sociedad de nuestros días.

Sin embargo, no basta asegurar el funcionamiento de un sistema que someta las actividades de construcción a la supervisión de los arqueólogos y establezca la financiación privada de las intervenciones. La cuestión clave es el adecuado encauzamiento de toda la actividad de intervención mediante una buena gestión del patrimonio histórico, que, entre otras cosas, minimice las intervenciones destructivas, potencie las soluciones alternativas y preventivas y rehúya el concepto de la excavación por la excavación. Además, la labor de gestión permitirá vincular la "arqueología de intervención", cuyo objetivo prioritario es la documentación y el salvamento de los recursos culturales afectados por actividades de construcción dentro de proyectos programados, con la investigación científica de problemas históricos concretos. Es obvio que la investigación histórica puede resultar notablemente beneficiada por la ampliación de la información disponible. 
En definitiva, concedemos un valor máximo a las actividades de gestión del patrimonio histórico como competencia primaria de la administración autonómica. Sin una adecuada gestión resulta imposible valorar los proyectos de mayor interés social o científico, racionalizar el empleo de fondos privados y públicos, conceder prioridades financieras y promover la promulgación de normativas y leyes de protección.

\section{NOTAS}

I El término sólo pretende definir a todos aquéllos arqueólogos cuyo ejercicio no se desarrolla en el marco de instituciones científicas u organismos públicos. Sustitúyase por arqueólogos de intervención u otro término similar, si se considera pertinente.

2 Para apreciar esta diversidad de los enfoques basta revisar los textos publicados por la Comisión de Arqueólogos del Colegio de Licenciados de Madrid.

3 Cfr. MENA MUÑOZ, P. y NOGUERAS MONTEAGUDO, E. (1990) "Excavaciones urbanas anteriores a 1985 y política arqueológica urbana de la Comunidad de Madrid" en Madrid del siglo IX al XI. Cat. Exposición, pp. 223-245. Madrid; NOGUERAS MONTEAGUDO, E. y MENA MUÑOZ, P. (1990) "Las excavaciones arqueológicas en el casco urbano de Madrid", op. cit. pp. 247-259 y MENA MUÑOZ, P. (|99|) "Arqueología urbana en el término municipal de Madrid". Arqueología, Paleontología y Etnografia I, pp. 203-216.

4 Por el mero hecho de que la empresa de arqueología contrate a un arqueólogo por menos de lo que aporta para su financiación la empresa que costea los trabajos. Tales procedimientos de extracción de plusvalía ya han planteado demandas, contra alguna de las más conocidas empresas, de actitudes netamente centradas en el negocio fácil ante el Colegio de Doctores y $\mathrm{R}$ senciados de Madrid.

5 Comisión de Arqueólogos CDL de Madrid/F. Velasco Steigrad: "Modelo de Gestión y desarrollo profesional en la Comunidad de Madrid", comunicación a la / Reunión de Arqueología Teórica, Santiago de Compostela, 1992, p. 8 y p. 10

6 DOMINGUEZ ALONSO, R. Ma; FERNÁNDEZ UGALDE, A.; HERCE YUSTE, J.L;; MENASANCH DE TOBARUELA, M.; MORENO LETE, E.; PRESAS VIAS, M. y VIGIL-ESCALERA, A. I ÁREA Sociedad
Cooperativa: "Arqueología Profesional e Intervención. Reflexiones desde una experiencia Cooperativa". Comunicación presentada en la / Reunión de Arqueología Teórica. Santiago de Compostela, 1.992. La categoría jurídica de AREA es la "Cooperativa de Trabajo Asociado".

7 Cfr. Comunicación a la / RAT de Santiago de Compostela, cit. en Nota 6.

8 Por otra parte, es sabido que la financiación por parte de la constructora es una recomendación del Consejo de Europa (Recomendación a los Estados miembros relativa a la protección y puesta en valor del patrimonio arqueológico en el contexto de las operaciones urbanísticas de ámbito urbano y rural, CDPH (89) 22. Estrasburgo, 13/4/1989). De hecho, en Madrid la financiación de la intervenciones arqueológicas por sus promotores no ha constituido nunca un obstáculo importante, y ello a pesar de no estar amparada por una normativa legal: simplemente, los promotores prefieren hacerse cargo de los costes para obtener sus peritajes en plazos razonables (si rechazaran hacerse cargo del peritaje, deberían esperar a que lo efectuara directamente la administración cuando dispusiera de fondos y de medios, caso hasta el momento no presentado, pues prima ante todo la rapidez del procedimiento). Han sido otras las causas del deterioro del sistema y de la calidad de las intervenciones: la competencia entre los propios arqueólogos y la escasa supervisión de los proyectos, procedimientos y resultados por la Consejería de Educación y Cultura.

9 Rechazamos absolutamente la afirmación, planteada en alguna ocasión en Madrid, de que la libertad del arqueólogo se extiende hasta la elección del procedimiento de excavación que considere más adecuado. Consideramos una exigencia ineludible que la metodología de excavación sea estratigráfica. Resulta paradójico que el método de excavación más empleado actualmente en Madrid sea todavía el de niveles artificiales. No hace falta comentar la destrucción que tales procedimientos arcaicos provocan en ambientes urbanos.

10 Como mencionábamos más arriba, se han llegado a establecer categorias como especialista en arqueología urbana versus especialista en arqueología de campo, o especialista en prospección... En la práctica, se trataba de institucionalizar el espacio de poder de los arqueólogos que venían trabajando desde un principio en la arqueología de intervención, y de cerrar el camino a las nuevas promociones. Como puede apreciarse, el corporativismo y la delimitación de cotos cerrados no son patrimonio exclusivo de las universidades. El modelo de Madrid está empezando a extenderse, y se discute su implantación en otras comunidades. 